\title{
Improved flu screening needed at airports
}

A irports in Toronto, Ontario, and Vancouver, British Columbia, act as major gateways for infectious diseases, and the federal government must better manage health risks arising from international air travel, warns a landmark report prepared for the Public Health Agency of Canada (PHAC).

Prescribed criteria for declaring travel bans, extensive use of thermal sensing technologies at airports, improved passenger screening and enhanced use of infectious disease polymerase chain reaction (PCR) testing are among the potential measures the report's authors suggest be investigated. Crafted by the Centre for Research on Inner City Health at St. Michael's Hospital in Toronto, the report is generating praise from the World Health Organization (WHO) and led the Kingdom of Saudi Arabia to recruit its authors to help it prepare for several million pilgrims to Mecca in November.

"We should be thinking much more closely about the role played by air travel in the international spread of infectious diseases and this study provides the evidence basis for surgical strikes on infectious disease transmission at key transmission points," says report coauthor Dr. Michael Gardam, director of infectious disease prevention and control at the Ontario Agency for Health Protection and Promotion.

Noting that measures like travel bans and thermal screening remain highly controversial and require refinement, coauthor Dr. Kamran Khan says pandemic response measures governing travel should direct "finite resources to high yield locations when epidemics arise, but more importantly, before epidemics occur. Like it or not, we live in a highly interconnected world. Our work just seeks to understand the rules that govern global travel and use them to our advantage."

The report, An Analysis of Canada's Vulnerability to Emerging Infectious

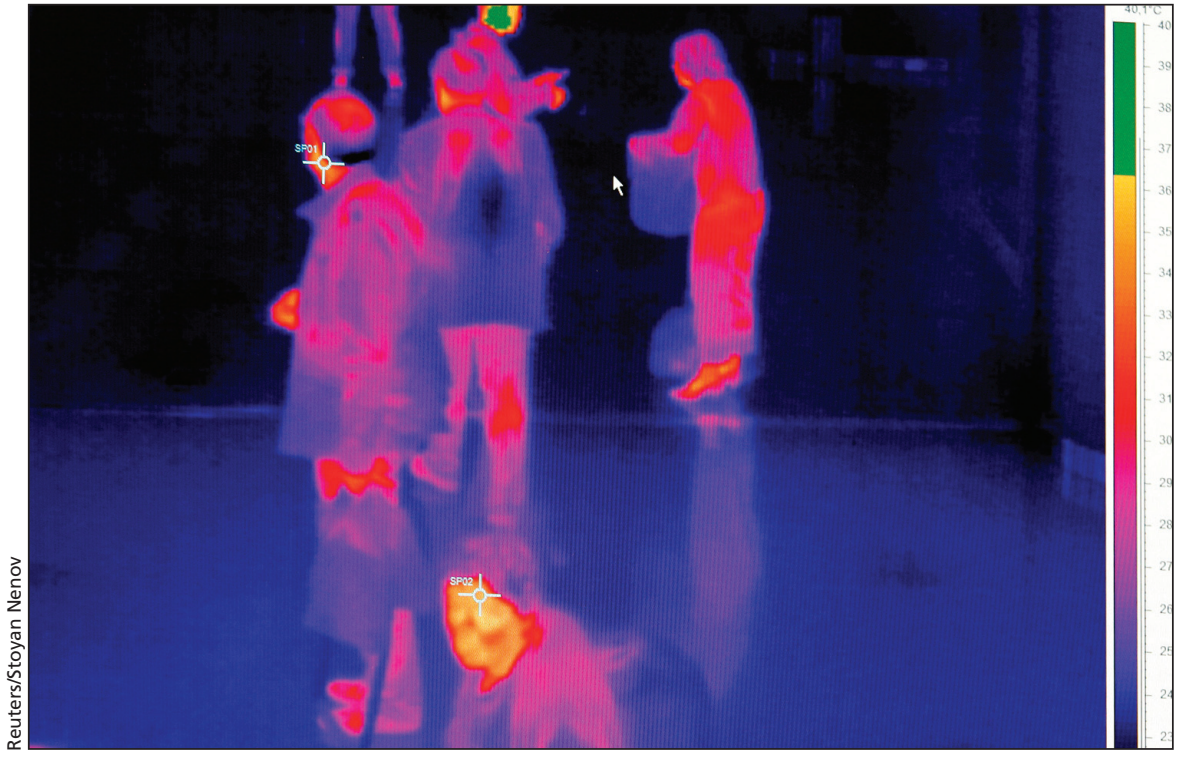

An airport thermal camera system shows the body heat of arriving passengers.

Disease Threats via the Global Airline Transportation Network, presents a mass of previously undisclosed international aviation data revealing that Toronto is the most vulnerable point of entry in Canada "ranking alongside the world's most centrally located cities within the global airline transportation network" (www.biodiaspora.com).

Within Canada, the airport in Richmond, BC, which serves Vancouver, represents the next greatest concern for the transmission of infectious diseases, followed by the Trudeau airport in Montréal, Quebec.

The St. Michael's team recommends the Canadian Pandemic Influenza Plan be amended to better reflect the role played by air travel in the spread of infectious diseases.

Under WHO regulations governing air travel and disease transmission, the Canadian government has designated entry points as subject to a set of rules aimed at encouraging countries to bolster planning for measures such as the comprehensive screening of travellers, the introduction of travel restrictions and the strengthening of international coordination of travel-related disease control.
But the report's coauthors say more must be done.

Canada needs to start working more closely with disease control authorities abroad, especially in countries with weak public health systems and high volumes of air passengers travelling to Canada, says Khan, who designed the multidisciplinary study.

"Diseases move very rapidly every day around the globe and we need to understand this in a routine way. Otherwise you're left responding when it arrives on your doorstep," Khan argues. "The best way to try to prevent diseases from travelling to Canada is to become more engaged with the countries we're connected with. Self-interest is a very compelling motivator to start addressing problems beyond our own borders. If you focus on maybe six places in the world, you can reduce $70 \%$ of your risks."

Khan and Gardam say their study which uses data obtained from prolonged negotiations with aviation industry officials and taps the expertise of specialists on aviation networks, disease modelling, cartography and statistics — was commissioned by PHAC 
after the severe acute respiratory syndrome (SARS) epidemic put a spotlight on aviation. "The overwhelming number of SARS cases that moved from country to country were by air travel," says Khan. "Now we're seeing the same with novel H1N1."

Although their study was completed shortly before pandemic (H1N1) 2009 emerged in Mexico in March 2008, it identifies Mexico as a primary source of disease spread by travellers to Canada, along with China, India and the Philippines.

"Mexico generates the second largest volume of international passenger traffic entering Canada from any developing country after China," the report notes. Almost 190000 air passengers arrive in Canada from Mexico City annually, a figure that almost doubles when travellers from Mexican resort destinations such as Cancun are added to the national total.

"After Hong Kong, Mexico City is the largest urban source of air traffic into Canada from a developing country," the study found. "Poverty, high population density, limited health care resources, coupled with a high volume of international inbound traffic to Canada provide conditions that could make Mexico City an important future source location."

Khan and Gardam's team have also noted that in March and April 2008 a total of 2.35 million passengers flew from Mexico to 1018 cities in 164 countries, with $80.7 \%$ of their destinations in the United States or Canada ( $N$ Engl J Med 2009;361:212-14[letter]).

The international destinations of travellers departing from Mexico in 2008 show a strong correlation to confirmed H1N1 importations associated with travel to Mexico in 2009, the authors stated in their letter to the New England Journal of Medicine. "Of the 20 countries worldwide with the highest volumes of international passengers arriving from Mexico, 16 had confirmed importations associated with travel to Mexico as of May 25, 2009."

The attempt to empirically map and simulate the linkages between air travel and the international spread of infectious diseases is important, says John Brownstein, an infectious disease surveillance specialist with the Children's Hospital Informatics Program at the Harvard-MIT Division of Health Sciences and Technology.

"It's understood that air travel plays a role," says Brownstein, whose work has linked air travel to the spread of influenza domestically in the US. "But surprisingly, this has never been shown even though it's so intuitively obvious. It's a very difficult thing to show. I think the tools that Khan and his team have assembled to do this are very exciting. This helps us work toward very targeted, scientifically driven measures beyond travel bans, which are so difficult because the amount of travel you may have to shut down is so extensive.

Pierre Formenty, emerging and dangerous pathogens team leader with the WHO's Global Alert and Response Department in Geneva, credits the study with resolving a key question that has puzzled many.

"If you look at the simulations for influenza in this study you will see that, conversely to what many people have said, Africa is not that strongly affected," he notes. "That's probably because there are not so many flights to and from and within Africa." As Khan and Gardam's report notes, developing countries where volumes of air travel have rapidly grown - such as Mexico and China - are of greatest concern.

Although Formenty says the $\$ 500000$ study makes it clear that the role of air travel warrants far greater attention among disease surveillance specialists, response thus far from the federal government has been muted.

PHAC spokeswoman Caroline Grondin said in an email that the study is "timely because of the outbreak of H1N1 influenza virus." She added that it is "being reviewed very carefully by officials involved in responding to outbreaks of infectious diseases" as updates are considered for Canada's pandemic influenza plan. - Paul Webster, Toronto, Ont.

DOI:10.1503/cmaj.109-3053 